\title{
The glucocorticoid receptor and cortisol levels in pediatric septic shock
}

\author{
Matthew N. Alder, Amy M. Opoka and Hector R. Wong ${ }^{*}$
}

\begin{abstract}
Background: There is controversy around the prescription of adjunct corticosteroids in patients with fluid-refractory septic shock, and studies provide mixed results, showing benefit, no benefit, and harm. Traditional means for evaluating whether a patient receives corticosteroids relied on anecdotal experience or measurement of serum cortisol production following stimulation. We set out to measure both serum cortisol and the intracellular signaling receptor for cortisol, the glucocorticoid receptor (GCR), in this group of patients.

Methods: We enrolled pediatric patients admitted to the pediatric intensive care unit with a diagnosis of systemic inflammatory response syndrome (SIRS), sepsis, or septic shock as well as healthy controls. We measured serum cortisol concentration and GCR expression by flow cytometry in peripheral blood leukocytes on the day of admission and day 3.

Results: We enrolled 164 patients for analysis. There was no difference between GCR expression comparing SIRS, sepsis, and septic shock. When all patients with septic shock were compared, those patients with a complicated course, defined as two or more organ failures at day 7 or death by day 28 , had lower expression of GCR in all peripheral blood leukocytes. Further analysis suggested that patients with the combination of low GCR and high serum cortisol had higher rates of complicated course (75\%) compared with the other three possible combinations of GCR and cortisol levels: low GCR and low cortisol (33\%), high GCR and high cortisol (33\%), and high GCR and low cortisol (13\%; $P<0.05)$.

Conclusions: We show that decreased expression of the GCR correlated with poor outcome from septic shock, particularly in those patients with high serum cortisol. This is consistent with findings from transcriptional studies showing that downregulation of GCR signaling genes portends worse outcome.
\end{abstract}

Keywords: Sepsis, Septic shock, Glucocorticoid receptor, Cortisol

\section{Background}

Pediatric septic shock remains a major cause of morbidity and mortality throughout the world [1]. Even with the highest level of care, many children succumb to septic shock despite years of research and millions of dollars spent. Heterogeneity among patients with septic shock has resulted in our inability to clearly show beneficial interventions beyond antibiotics and supportive care. This is nowhere more obvious than in our current practice of prescribing adjunctive corticosteroids for patients with refractory septic shock. In an era of precision medicine, we need tools that allow us to better classify patients with septic shock to allow evidence-based practice rather than anecdotal experience.

\footnotetext{
* Correspondence: hector.wong@cchmc.org

Division of Critical Care Medicine, Cincinnati Children's Hospital Medical Center, Children's Hospital Research Foundation, 3333 Burnet Avenue, MLC 2005, Cincinnati, OH 45229, USA
}

Whether or not to prescribe corticosteroids for patients with fluid-refractory septic shock has been questioned for more than four decades, and no definitive answer currently exists. Many reviews have summarized these studies [2-5]. Some studies have suggested benefit [6], but subsequent trials have not been able to confirm these findings [7], whereas other studies suggest that there may be harm due to administration of corticosteroids [8-11]. This was further reiterated with two recent large studies published side by side in the New England Journal of Medicine, again showing conflicting results [12, 13]. Heterogeneity within the septic shock population undoubtedly plays into our confusion in trial results, as patients present with different pathophysiology, pathogens, and comorbidities. Until a more definitive trial can better categorize patients into more than just septic shock or no septic shock, we are unlikely to be able to resolve this question. 
For some time, our group has focused on the transcriptional characteristics of pediatric patients with septic shock to attempt to de-convolute the heterogeneity of patients $[14,15]$. This approach has allowed the subclassification of patients into different risk strata and separate endotypes based on a 100-gene panel [16]. These studies have consistently shown that those patients with downregulation of genes in the glucocorticoid signaling pathway have worse outcomes and that corticosteroid prescription is associated with poor outcome in this group. It is unclear why these patients have downregulation of the glucocorticoid pathway or whether this downregulation directly contributes to poor outcomes.

The primary receptor for the glucocorticoid pathway is the glucocorticoid receptor (GCR), which exists in the cytoplasm of most cells and can influence expression of up to $20 \%$ of the genes in peripheral blood cells $[3,17$, 18]. The two predominant isoforms of the GCR are GCR alpha and GCR beta. Functionality of the GCR signaling axis comes primarily through the major isoform GCR alpha. The second isoform, GCR beta, is generated by the use of an alternative final exon and creates a dominant negative GCR. We sought to test the relationship between the primary signaling molecule of the glucocorticoid pathway, cortisol, and the primary receptor, GCR alpha, to test whether we could better understand regulation of the glucocorticoid axis in patients with septic shock.

\section{Methods}

\section{Prospective enrollment of study subjects and data collection}

The institutional review board of Cincinnati Children's Hospital Medical Center approved the protocol for the prospective collection of human blood samples and clinical data. Patients not older than 18 years of age admitted to the pediatric intensive care unit (PICU) and meeting pediatric-specific consensus criteria for systemic inflammatory response syndrome (SIRS), sepsis, or septic shock were eligible for enrollment. There were no exclusion criteria for enrollment. Legal guardians were approached for informed consent prior to data or sample collection. For control samples, children presenting for elective hernia repair were approached for willingness to donate blood at the time of intravenous catheter placement. All control subjects were screened to ensure good health and no febrile illness, corticosteroids, or anti-inflammatory medication in the prior two weeks.

"Day 1" samples were obtained within the first 24 hours of meeting criteria for SIRS, sepsis, or septic shock in the PICU; for most samples, this was at presentation to the PICU. "Day 3" samples were obtained 48 hours later. While patients were in the PICU, clinical and laboratory data were collected. Evidence for organ failure was tracked for up to 7 days by using previously published criteria
[19], except for mortality, which was tracked for 28 days after enrollment. Illness severity was estimated by using the Pediatric Risk of Mortality (PRISM) score, which is based on laboratory values and clinical variables at the time of enrollment.

\section{Flow cytometry}

Whole blood samples were stained in accordance with standard protocols used for intracellular staining of cytosolic proteins. Briefly, blood samples underwent red cell lysis using ACK buffer (Thermo Fisher, Waltham, MA, USA) followed by washing. Cells were blocked with $10 \%$ human serum in flow buffer-phosphate-buffered saline (PBS) with $1 \%$ bovine serum albumin (BSA)-and surface-stained with CD3, CD14, and CD66b (Becton Dickinson, Franklin Lakes, NJ, USA). After surface stain, cells were washed and fixed with $2 \%$ paraformaldehyde. Cells were washed and permeabilized with intracellular cytokine staining buffer (ICCS Buffer-10 mM HEPES, 0.1\% BSA, 0.1\% saponin, 0.1\% Azide in PBS). Cells were again blocked with $10 \%$ human serum and followed by staining for intracellular GCR alpha (Abcam, Cambridge, UK). Stained cells were analyzed on a Canto II flow cytometer (Becton Dickinson). To ensure comparable mean fluorescence intensity (MFI) for samples collected over a long period of time, each time a sample was run, Rainbow Calibration Particles (Becton Dickinson) were used to establish consistent voltage settings.

\section{Serum cortisol measurement}

Cortisol levels were measured from serum samples in the hospital's clinical lab by using the Access Cortisol Assay (Beckman Coulter, Brea, CA, USA) on the Access 2 immunoanalyzer.

\section{Statistical analysis}

Statistical procedures used SigmaStat Software (Systat Software, Inc., San Jose, CA, USA). Comparisons between groups used the Mann-Whitney $U$ test, rank sum test, chi-squared test, or Fisher's exact test, as appropriate. The association between GCR alpha expression and serum cortisol concentrations was evaluated by using linear regression and all available day 1 and day 3 paired data. The association between GCR alpha expression and outcome was modeled by using multivariable regression, adjusting for illness severity, exposure to corticosteroids, age, and comorbidity. The primary outcome variable for the regression procedures was a complicated course, defined as the persistence of two or more organ failures at day 7 of septic shock or 28-day mortality. Since this was an exploratory study, a priori we planned to extend our initial analysis, guided by the findings. For ease of reference, we describe exploratory analyses in the Results section. 


\section{Results}

Table 1 shows the clinical and demographic characteristics of the study cohort. Subjects with sepsis and septic shock were older than controls. Patients with septic shock had higher baseline cortisol concentrations, higher PRISM scores, and a higher rate of a complicated course. No other differences were noted.

Additional file 1 shows the GCR alpha MFI across all four study groups, for all cells combined, as well as subpopulations of leukocytes, lymphocytes, monocytes, neutrophils, on day 1 and day 3 of admission. GCR alpha expression was greater than that of controls for some combinations of cell types and study categories, but no consistent pattern was noted. GCR alpha expression was not different between subjects in the SIRS, sepsis, and septic shock groups.

Table 2 compares GCR alpha expression among the subjects with septic shock, grouped into those with and without a complicated course. No differences were noted on day 1. In contrast, on day 3, GCR alpha expression was significantly decreased among subjects with a complicated course. This was evident for all white blood cells as well as the individual white blood cell populations.

Based on the observation that day 3 GCR alpha expression was consistently decreased among patients with septic shock and a complicated course, we next used logistic regression to further assess the association between GCR alpha expression on all white blood cells and a complicated course. As shown in Table 3, by univariable analysis, higher day 3 GCR alpha expression was associated with decreased odds of a complicated course. Higher PRISM score and exposure to exogenous corticosteroids were associated with increased odds of a complicated course. Age and the presence of a comorbidity were not associated with a complicated course. We next conducted a multivariable analysis to determine whether day $3 \mathrm{GCR}$ alpha expression, PRISM score, and corticosteroid exposure were independently associated with a complicated course (Table 3). Although there were trends, none of the three variables was independently associated with a complicated course. This suggests co-linearity and complex interactions between GCR alpha expression, illness severity, and corticosteroid exposure. When we considered interaction terms between the three variables, none of the interaction terms was associated with a complicated course (data not shown).

We then sought to test whether there was a relationship between GCR alpha expression and serum cortisol concentration among patients with septic shock. When all patients, regardless of whether they received exogenous corticosteroids, were considered by linear regression, there was no association between serum cortisol concentration and GCR expression from all cells (Fig. $1 ; R=0.058, P=$ 0.532 ). This lack of association was also evident in the subgroup that received exogenous corticosteroids $(R=0.183, P$

Table 1 Demographic and clinical variables of the study cohort

\begin{tabular}{|c|c|c|c|c|}
\hline & Controls & SIRS & Sepsis & Septic shock \\
\hline $\bar{N}$ & 35 & 17 & 23 & 89 \\
\hline Median age, years (IQR) & $2.0(0.6-5.0)$ & $5.7(3.3-10.8)$ & $6.6(3.8-14.3)^{a}$ & $7.4(1.7-14.2)^{a}$ \\
\hline Males, $n(\%)$ & $24(69)$ & $8(47)$ & $11(48)$ & $53(60)$ \\
\hline Median baseline cortisol, $\mu \mathrm{g} / \mathrm{dL}$ (IQR) & $15(9-21)$ & $27(14-35)$ & $19(10-30)$ & $25(13-49)^{a}$ \\
\hline Mortality, $n(\%)$ & - & $2(12)$ & $0(0)$ & $6(8)$ \\
\hline Complicated course, n (\%) & - & $2(12)$ & $0(0)$ & $21(24)^{b}$ \\
\hline Median PRISM, (IQR) & - & $4(3-11)$ & $5(3-8)$ & $11(7-15)^{c}$ \\
\hline Gram-positive bacteria, $n(\%)$ & - & - & $10(43)$ & $15(17)$ \\
\hline Gram-negative bacteria, $n(\%)$ & - & - & $0(0)$ & $19(21)$ \\
\hline Other organism, $n(\%)^{d}$ & - & - & $0(0)$ & $8(9)$ \\
\hline Culture negative, $n$ (\%) & - & - & $13(57)$ & $47(53)$ \\
\hline Comorbidity, $n(\%)$ & - & $14(82)$ & $16(70)$ & $60(67)$ \\
\hline Malignancy, n (\%) & - & $0(0)$ & $3(13)$ & $14(16)$ \\
\hline Immunosuppression, $n(\%)^{e}$ & - & $1(6)$ & $3(13)$ & $20(22)$ \\
\hline Bone marrow transplantation, $n(\%)$ & - & $0(0)$ & $1(4)$ & $13(15)$ \\
\hline Received corticosteroids, $n(\%)$ & - & $2(12)$ & $7(30)$ & $37(42)$ \\
\hline
\end{tabular}

${ }^{a} P<0.05$ versus controls, analysis of variance (ANOVA) on ranks

${ }^{\mathrm{b}} P<0.05$, chi-squared, 2 degrees of freedom

${ }^{c} P<0.05$ versus systemic inflammatory response syndrome (SIRS) and sepsis, ANOVA on ranks

${ }^{d}$ Refers to viral, fungal, or mixed infections

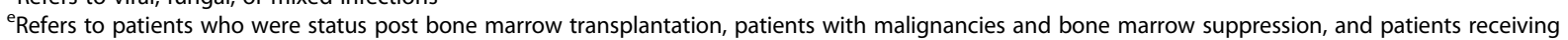
immunosuppression medications following solid organ transplantation.

Abbreviations: IQR interquartile range, PRISM Pediatric Risk of Mortality 
Table 2 Glucocorticoid receptor alpha expression table comparing patients with septic shock, with and without a complicated course

\begin{tabular}{llll}
\hline Cell type & Day & Non-complicated course & Complicated course \\
\hline All white blood cells & 1 & $1965(1080-2780)$ & $1830(1318-3506)$ \\
All white blood cells & 3 & $3019(1741-3474)$ & $1792(1224-2538)^{\mathrm{a}}$ \\
Lymphocytes & 1 & $878(441-1243)$ & $773(342-1182)$ \\
Lymphocytes & 3 & $1014(798-1619)$ & $714(304-1267)^{\mathrm{a}}$ \\
Monocytes & 1 & $3035(1954-3566)$ & $2495(1601-4525)$ \\
Monocytes & 3 & $3669(3264-4375)$ & $2557(1564-3388)^{\mathrm{a}}$ \\
Neutrophils & 1 & $2320(1474-3039)$ & $1981(1440-3600)$ \\
Neutrophils & 3 & $3497(2420-4062)$ & $2067(1518-2845)^{\mathrm{a}}$ \\
\hline
\end{tabular}

Shown as median mean fluorescence intensity (interquartile range). ${ }^{a} P<0.05$ versus non-complicated course, rank sum test

$=0.278)$ and the subgroup that did not receive exogenous corticosteroids $(R=0.264, P=0.051)$. In addition, this lack of association was evident when excluding patients with immunosuppression $(R=0.067, P=0.580)$. Since GCR expression and serum cortisol were not related or had only a weak trend (in the subgroup that did not receive corticosteroids), we next categorized patients into groups of high or low cortisol and high or low GCR expression. To do this, we used the median values of cortisol and GCR MFI for all patients with septic shock. Values above the median were considered "high expression", and values below the median were considered "low expression". Table 4 compares indices of illness severity among these four groups. Subjects with low serum cortisol and high GCR expression tended to be less severely ill. In contrast, subjects with high serum cortisol and low GCR expression on day 3 had a significantly higher rate of a complicated course, higher PRISM scores, fewer PICU-free days, and fewer vasopressor-free days. Among the 14 subjects with high serum cortisol and low GCR expression on day 1 who also had available day 3 data, nine remained in the high serum cortisol and low GCR expression group on day 3 and seven of these subjects (78\%) had a complicated course. Among the remaining subjects with high serum cortisol and low GCR expression on day 1, but who transitioned to one of the other three possible groups by day 3 , none had a complicated course.

\section{Discussion}

The current recommendation for patients with fluid- and vasopressor-refractory septic shock is to consider treatment with corticosteroids [20, 21]. However, there is a lack of consensus and conclusive evidence that corticosteroids are beneficial to all pediatric patients with septic shock. We postulated that evaluating serum cortisol alone or the response to stimulation with adrenocorticotropic hormone $(\mathrm{ACTH})$ was insufficient to predict which patients would respond to corticosteroids. As a first step toward testing this hypothesis, we characterized a cohort of pediatric patients and tested expression of both cortisol and the intracellular GCR. This hypothesis was based on our previous findings that patients with downregulation of the glucocorticoid signaling genes had worse outcomes.

GCR alpha measurement in this patient cohort did not show any consistent patterns when comparing patients with different diagnoses, SIRS, sepsis, or septic shock. Some white blood cell populations, such as lymphocytes, did show increased GCR expression with any diagnosis at the time of admission, but no other reliable trend was present (Additional file 1). Further subclassification of patients with septic shock into those with a complicated and non-complicated course revealed that those patients who had a complicated course had lower expression of GCR alpha. Admission samples tended to be lower in those

Table 3 Univariable and multivariable logistic regression to test for associations between the listed variables and a complicated course

\begin{tabular}{|c|c|c|c|c|c|c|}
\hline \multirow[t]{2}{*}{ Variable } & \multicolumn{3}{|c|}{ Univariable analysis } & \multicolumn{3}{|c|}{ Multivariable analysis } \\
\hline & Odds ratio & $95 \% \mathrm{Cl}$ & $P$ value & Odds ratio & $95 \% \mathrm{Cl}$ & $P$ value \\
\hline Day 3 GCR alpha MFI & 0.03 & 0.00 to 0.85 & 0.039 & 0.049 & 0.00 to 2.12 & 0.117 \\
\hline PRISM & 1.3 & 1.1 to 1.5 & 0.002 & 1.2 & 1.0 to 1.4 & 0.058 \\
\hline Corticosteroids & 4.8 & 1.4 to 16.2 & 0.012 & 3.7 & 0.8 to 16.8 & 0.092 \\
\hline Age & 1.0 & 0.9 to 1.1 & 0.454 & - & - & - \\
\hline Comorbidity & 2.0 & 0.5 to 7.4 & 0.317 & - & - & - \\
\hline Bone marrow transplantation & 1.6 & 0.6 to 4.3 & 0.393 & - & - & - \\
\hline Immunosuppression & 0.4 & 0.3 to 2.1 & 0.640 & & & \\
\hline
\end{tabular}

Abbreviations: $C l$ confidence interval, GCR glucocorticoid receptor, MFI mean fluorescence intensity, PRISM Pediatric Risk of Mortality 


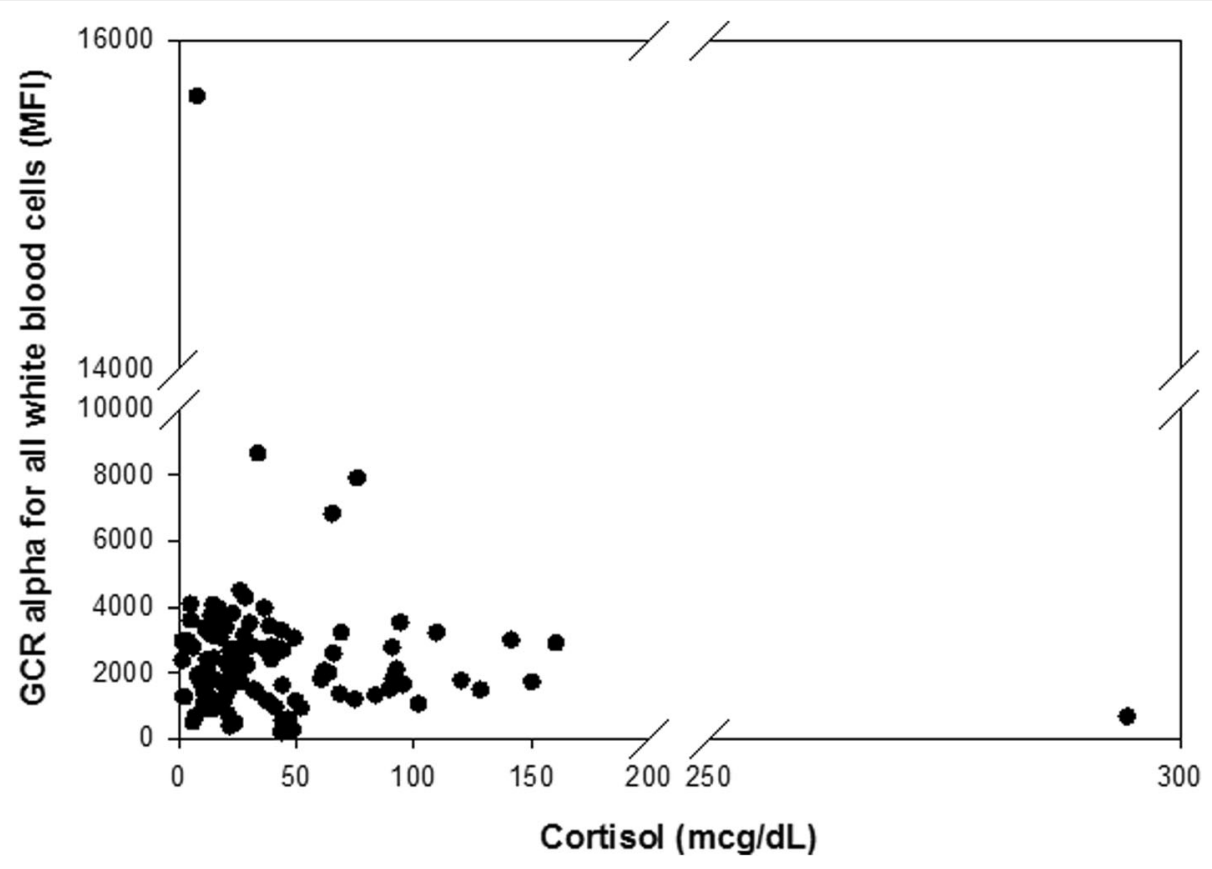

Fig. 1 No correlation between glucocorticoid receptor (GCR) alpha and serum cortisol. Comparison of flow cytometric mean fluorescence intensity (MFI) of all leukocyte GCR alpha expression relative to serum cortisol $(R=0.058, P=0.532)$

patients with a complicated course but this became significant only at day 3 (Table 2). Univariate analysis further confirmed that high expression of GCR alpha decreased the odds of a complicated course (Table 3). This finding is consistent with our previous transcriptional studies showing that patients with a complicated course had downregulation of genes in the glucocorticoid signaling pathway $[11,16]$. Other groups have also shown reduced GCR alpha expression in pediatric patients critically ill with septic shock [22].

We found no correlation between serum cortisol level and the GCR alpha receptor. This suggests that these two important molecules are subject to independent regulation during critical illness. For this reason, we

Table 4 Indices of illness severity among the cortisol high/low and glucocorticoid receptor alpha high/low groups

\begin{tabular}{|c|c|c|c|c|}
\hline & High/High & High/Low & Low/High & Low/Low \\
\hline \multicolumn{5}{|c|}{ Day 1 relative cortisol level/relative GCR alpha level } \\
\hline Number & 20 & 26 & 13 & 30 \\
\hline Rate of complicated course & $30 \%$ & $27 \%$ & $8 \%$ & $23 \%$ \\
\hline Median PRISM, (IQR) & $13(8-19)$ & $13(7-18)$ & $9(4-11)$ & $10(7-14)$ \\
\hline Maximum organ failures, median (IQR) & $2(2-3)$ & $2(1-3)$ & $2(1-3)$ & $2(1-2)$ \\
\hline Median PICU-free days, (IQR) & $20(15-26)$ & $23(9-26)$ & $24(21-27)$ & $24(20-26)$ \\
\hline Median vasopressor-free days, (IQR) & $26(22-27)$ & $26(23-27)$ & $27(25-27)$ & $26(25-27)$ \\
\hline \multicolumn{5}{|c|}{ Day 3 relative cortisol level/relative GCR alpha level } \\
\hline N & 12 & 12 & 15 & 12 \\
\hline Rate of complicated course & $33 \%$ & $75 \%{ }^{\mathrm{a}}$ & $13 \%$ & $33 \%$ \\
\hline Median PRISM, (IQR) & $13(10-14)$ & $20(14-24)^{b}$ & $8(6-12)$ & $10(8-14)$ \\
\hline Maximum organ failures, median (IQR) & $2(2-3)$ & $3(2-4)^{b}$ & $2(2-2)$ & $2(2-2)$ \\
\hline Median PICU-free days, (IQR) & $20(17-25)$ & $0(0-13)^{c}$ & $21(10-25)$ & $22(9-25)$ \\
\hline Median vasopressor-free days, (IQR) & $25(23-27)$ & $18(0-24)^{c}$ & $25(25-27)$ & $25(24-26)$ \\
\hline
\end{tabular}

${ }^{a} P<0.05$, chi-squared, 3 degrees of freedom

${ }^{\mathrm{b}} P<0.05$, versus low/high and low/low groups, analysis of variance (ANOVA) on ranks

${ }^{c} P<0.05$, versus all other groups, ANOVA on ranks

Abbreviations: GCR glucocorticoid receptor, IQR interquartile range, PICU pediatric intensive care unit, PRISM Pediatric Risk of Mortality 
further subdivided patients into groups with high and low expression of cortisol and GCR alpha. This method of grouping patients yielded two interesting findings. First, those patients with low cortisol levels and high GCR expression on day 1 or day 3 had the lowest rates of complicated course. This finding seems consistent with patients with an overall low level of stress response to infection demonstrated by low cortisol level and high GCR alpha receptor available for adequate signaling. The group with the exact opposite levels, high cortisol and low GCR expression, had the worst outcomes with much higher rates of complicated course (Table 4) and this association appeared to be strongest among those who persisted with high cortisol and low GCR expression from day 1 to day 3 . This finding suggests a subgroup of patients with persistent high levels of physiological and biological stress, as indicated by high production of cortisol, who have concomitant low expression of the GCR and therefore may be unable to adequately respond to the stress signal. If this is true, then it would be difficult to conceive how prescription of corticosteroids could be of benefit for this subgroup.

We choose to focus on GCR alpha because it is the primary signaling molecule of the glucocorticoid axis. We also evaluated GCR beta, but low overall expression and inconsistent intracellular staining in peripheral blood cells prevented meaningful interpretation of the data. In future studies, characterization of GCR beta may further clarify those patients who will not benefit from corticosteroid treatment as those cells with high GCR beta would be expected not to respond to glucocorticoid treatment as GCR beta is a dominant negative receptor $[23,24]$.

A weakness of this study is that we were able to measure GCR expression only in peripheral blood cells, which may not be the most important cells for determining the physiologic effects of cortisol on and the glucocorticoid signaling axis. Evaluating GCR expression in tissue, particularly vasculature, may be more meaningful, but inaccessibility is a major impediment. Few studies have looked at tissue expression of GCR alpha and beta [25]; however, these studies are also complicated by many cell types, and differing levels of GCR expression make meaningful interpretation difficult. In addition, not all of the patients with septic shock had an available day 3 sample because they were discharged from the PICU and no longer had vascular access for the study blood draws. This raises the possibility of unintended selection bias. Finally, we collected samples as soon as possible after admission to the PICU to attempt to measure levels as close as possible to the diagnosis of septic shock. However, this resulted in samples being collected throughout the day. The potential for variation in cortisol levels on the basis of circadian rhythm is not accounted for in this approach.

\section{Conclusions}

We have shown differential expression of two of the major glucocorticoid signaling molecules-cortisol and GCR alpha-in critically ill children. The finding of low expression of GCR in patients with a complicated course matches previous studies showing that patients with downregulation of this axis have worse outcomes. These studies may account for why the ACTH stimulation test alone does not explain which patients will benefit from corticosteroids as the ability to generate an appropriate level of cortisol during stress also requires a receptor for the cortisol to have an effect. Some patients with low GCR expression already have high serum cortisol, calling into question whether exogenous corticosteroids will be of any benefit to this group of patients. On the other hand, there are patients with high GCR expression but low cortisol who may very well benefit from additional corticosteroids. Future studies will need to look at GCR expression in specific diseases that have more consistently shown benefit from treatment with corticosteroids, such as dengue fever. The ultimate answer may not come until we are able to study these subgroups of patients in a clinical trial. As momentum builds for a randomized pediatric trial testing the role of corticosteroids in septic shock, perhaps evaluating intracellular expression of GCR would be beneficial.

\section{Additional file}

Additional file 1: Glucocorticoid receptor (GCR) alpha expression table for all study groups. Shown as median mean fluorescence intensity (MFI) (interquartile range, or IQR). Contains GCR alpha MFI data for all patients evaluated in the cohort. Includes evaluating all cells together as well as individual cell populations. (DOCX $13 \mathrm{~kb}$ )

\section{Abbreviations}

ACTH: Adrenocorticotropic hormone; GCR: Glucocorticoid receptor; MFI: Mean fluorescence intensity; PICU: Pediatric intensive care unit; PRISM: Pediatric risk of mortality; SIRS: Systemic inflammatory response syndrome

\section{Acknowledgments}

We thank Erin Frank, Kelli Howard, Toni Yunger, and Laura Benken for obtaining samples and families' consent and the Cincinnati Children's Hospital Research Flow Cytometry Core for assistance.

\section{Funding}

Funding was provided by the National Institute of General Medical Sciences (grants MNA K08GM124298, HRW R35GM126943, and R01GM108025).

\section{Availability of data and materials}

The datasets used or analyzed (or both) during the current study are available from the corresponding author on reasonable request.

\section{Authors' contributions}

MNA and HRW generated the hypothesis, designed the experiments, interpreted the data, and wrote the manuscript. AMO conducted the experiments, interpreted the data, and edited the manuscript. All authors read and approved the final manuscript. 


\section{Ethics approval and consent to participate}

The institutional review board at Cincinnati Children's Hospital reviewed and approved the study protocol. Biological samples and clinical data were gathered after signed informed consent by the parents or legal guardians of the study subjects was obtained.

\section{Consent for publication}

We consent for publication in Critical Care

\section{Competing interests}

The authors declare that they have no competing interests.

\section{Publisher's Note}

Springer Nature remains neutral with regard to jurisdictional claims in published maps and institutional affiliations.

Received: 24 May 2018 Accepted: 4 September 2018

Published online: 29 September 2018

\section{References}

1. Watson RS, Carcillo JA, Linde-Zwirble WT, Clermont G, Lidicker J, Angus DC. The epidemiology of severe sepsis in children in the United States. Am Respir Crit Care Med. 2003;167:695-701.

2. Annane D, Bellissant E, Bollaert PE, Briegel J, Confalonieri M, De Gaudio R, et al. Corticosteroids in the treatment of severe sepsis and septic shock in adults: a systematic review. JAMA. 2009:301:2362-75.

3. Zimmerman JJ. Adjunctive steroid therapy for treatment of pediatric septic shock. Pediatr Clin N Am. 2017;64:1133-46.

4. Annane D, Bellissant E, Bollaert PE, Briegel J, Keh D, Kupfer Y. Corticosteroids for treating sepsis. Cochrane Database Syst Rev. 2015:CD002243.

5. Volbeda M, Wetterslev J, Gluud C, Zijilstra JG, van der Horst IC, Keus F. Glucocorticosteroids for sepsis: systematic review with meta-analysis and trial sequential analysis. Intensive Care Med. 2015:41:1220-34.

6. Annane D, Sebille V, Charpentier C, Bollaert PE, Francois B, Korach JM, et al. Effect of treatment with low doses of hydrocortisone and fludrocortisone on mortality in patients with septic shock. JAMA. 2002;288:862-71.

7. Sprung CL, Annane D, Keh D, Moreno R, Singer M, Freivogel K, et al. Hydrocortisone therapy for patients with septic shock. N Engl J Med. 2008; 358:111-24

8. Markovitz BP, Goodman DM, Watson RS, Bertoch D, Zimmerman J. A retrospective cohort study of prognostic factors associated with outcome in pediatric severe sepsis: what is the role of steroids? Pediatr Crit Care Med. 2005;6:270-4.

9. Zimmerman JJ, Williams MD. Adjunctive corticosteroid therapy in pediatric severe sepsis: observations from the RESOLVE study. Pediatr Crit Care Med. 2011;12:2-8

10. Wong HR, Cvijanovich NZ, Allen GL, Thomas NJ, Freishtat RJ, Anas N, et al. Corticosteroids are associated with repression of adaptive immunity gene programs in pediatric septic shock. Am J Respir Crit Care Med. 2014;189: 940-6.

11. Wong HR, Cvijanovich NZ, Anas N, Allen GL, Thomas NJ, Bigham MT, et al. Endotype transitions during the acute phase of pediatric septic shock reflect changing risk and treatment response. Crit Care Med. 2018;46:e242-9.

12. Annane D, Renault A, Brun-Buisson C, Megarbane B, Quenot JP, Siami S, et al. Hydrocortisone plus fludrocortisone for adults with septic shock. N Engl J Med. 2018;378:809-18

13. Venkatesh B, Finfer S, Cohen J, Rajbhandari D, Arabi Y, Bellomo R, et al. Adjunctive glucocorticoid therapy in patients with septic shock. N Engl J Med. 2018:378:797-808.

14. Wong HR, Cvijanovich N, Allen GL, Lin R, Anas N, Meyer K, et al. Genomic expression profiling across the pediatric systemic inflammatory response syndrome, sepsis, and septic shock spectrum. Crit Care Med. 2009;37:1558-66.

15. Wong HR, Cvijanovich N, Lin R, Allen GL, Thomas NJ, Willson DF, et al. Identification of pediatric septic shock subclasses based on genome-wide expression profiling. BMC Med. 2009;7:34.

16. Wong HR, Cvijanovich NZ, Anas N, Allen GL, Thomas NJ, Bigham MT, et al. Developing a clinically feasible personalized medicine approach to pediatric septic shock. Am J Respir Crit Care Med. 2015;191:309-15.

17. Galon J, Franchimont D, Hiroi N, Frey G, Boettner A, Ehrhart-Bornstein M, et al. Gene profiling reveals unknown enhancing and suppressive actions of glucocorticoids on immune cells. FASEB J. 2002;16:61-71.
18. Koper JW, van Rossum EF, van den Akker EL. Glucocorticoid receptor polymorphisms and haplotypes and their expression in health and disease. Steroids. 2014;92:62-73.

19. Goldstein B, Giroir B, Randolph A, International Consensus Conference on Pediatric S. International pediatric sepsis consensus conference: definitions for sepsis and organ dysfunction in pediatrics. Pediatr Crit Care Med. 2005;6:2-8.

20. Rhodes A, Evans LE, Alhazzani W, Levy MM, Antonelli M, Ferrer R, et al. Surviving Sepsis campaign: international guidelines for Management of Sepsis and Septic Shock: 2016. Crit Care Med. 2017:45:486-552.

21. Dellinger RP, Levy MM, Rhodes A, Annane D, Gerlach H, Opal SM, et al. Surviving sepsis campaign: international guidelines for management of severe sepsis and septic shock: 2012. Crit Care Med. 2013;41:580-637.

22. van den Akker EL, Koper JW, Joosten K, de Jong FH, Hazelzet JA, Lamberts SW, et al. Glucocorticoid receptor mRNA levels are selectively decreased in neutrophils of children sepsis. Intensive Care Med. 2009:35:1247-54.

23. Guerrero J, Gatica HA, Rodriguez M, Estay R, Goecke IA. Septic serum induces glucocorticoid resistance and modifies the expression of glucocorticoid isoforms receptors: a prospective cohort study and in vitro experimental assay. Crit Care. 2013;17:R107.

24. Cohen J, Pretorius CJ, Ungerer JP, Cardinal J, Blumenthal A, Presneill J, et al. Glucocorticoid sensitivity is highly variable in critically ill patients with septic shock and is associated with disease severity. Crit Care Med. 2016:44:1034-41.

25. Goldbart AD, Veling MC, Goldman JL, Li RC, Brittian KR, Gozal D. Glucocorticoid receptor subunit expression in adenotonsillar tissue of children with obstructive sleep apnea. Pediatr Res. 2005;57:232-6.
Ready to submit your research? Choose BMC and benefit from:

- fast, convenient online submission

- thorough peer review by experienced researchers in your field

- rapid publication on acceptance

- support for research data, including large and complex data types

- gold Open Access which fosters wider collaboration and increased citations

- maximum visibility for your research: over $100 \mathrm{M}$ website views per year

At $\mathrm{BMC}$, research is always in progress.

Learn more biomedcentral.com/submissions 